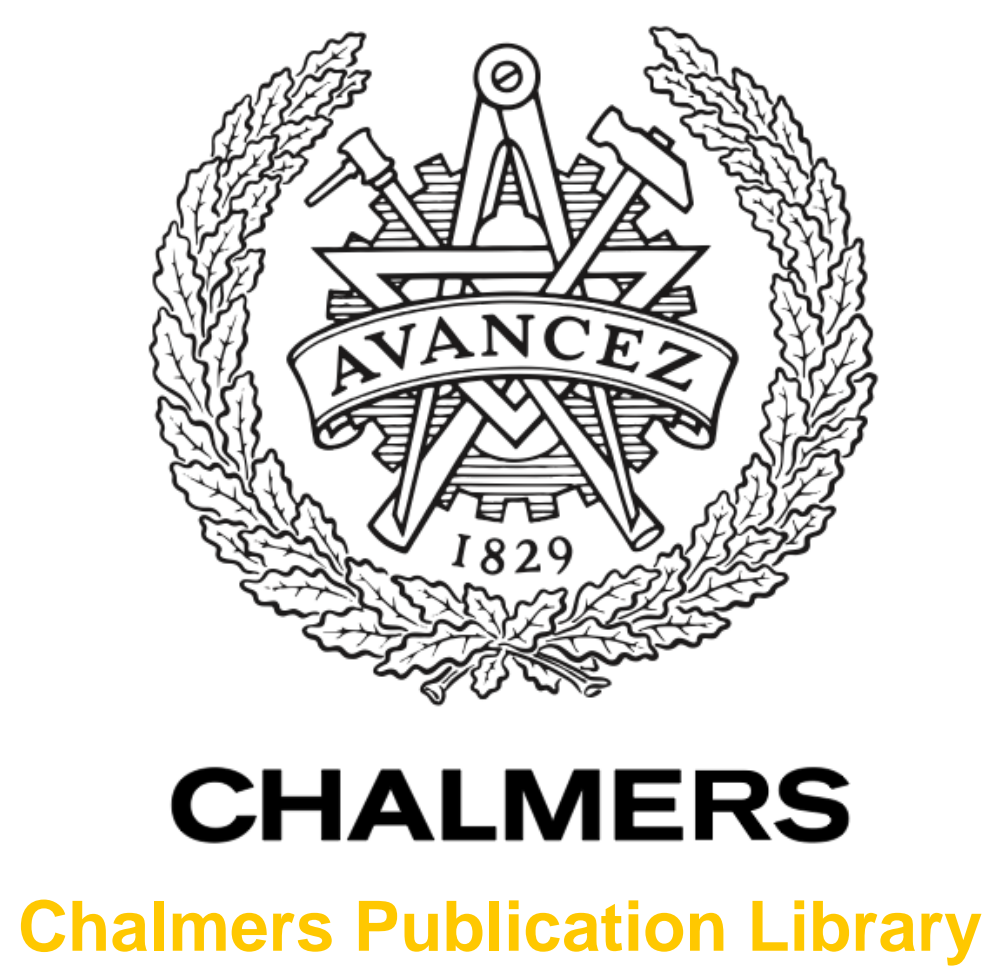

\title{
Optical Arrangements for Time-Gated Ballistic Imaging
}

This document has been downloaded from Chalmers Publication Library $(\mathrm{CPL})$. It is the author's version of a work that was accepted for publication in:

Imaging and Applied Optics 2014

Citation for the published paper:

Rahm, M. ; Sedarsky, D. ; Linne, M. (2014) "Optical Arrangements for Time-Gated Ballistic Imaging". Imaging and Applied Optics 2014

http://dx.doi.org/10.1364/ISA.2014.IM4C.5

Downloaded from: http://publications.lib.chalmers.se/publication/200445

Notice: Changes introduced as a result of publishing processes such as copy-editing and formatting may not be reflected in this document. For a definitive version of this work, please refer to the published source. Please note that access to the published version might require a subscription. 


\title{
Optical Arrangements for Time-Gated Ballistic Imaging
}

\author{
M. Rahm ${ }^{1, *}$, M. Paciaroni ${ }^{2}$, Z. Wang ${ }^{3}$, D. Sedarsky ${ }^{1}$, M. Linne ${ }^{1}$ \\ ${ }^{1}$ Department of Applied Mechanics, Chalmers University of Technology, SE-412 96 Gothenburg, Sweden \\ ${ }^{2}$ Department of Physics \& Engineering, Fort Lewis College, Durango, Colorado 81301, USA \\ ${ }^{3}$ Combustion Physics, Lund University, P.O. Box 118, SE-221 00 Lund, Sweden \\ *Authore-mail address: mattias.rahm@chalmers.se
}

\begin{abstract}
We report on a comparison of two optical setups used in time-gated ballistic imaging simulating monodisperse scattering environments with polystyrene spheres in different sizes and concentrations suspended in water.
\end{abstract}

OCIS codes: (110.0113) Imaging through turbid media, (190.3270) Kerr effect.

\section{Introduction}

Fuel sprays and the conversion of liquid fuel to combustible fuel vapor are critical to the performance of several types of combustion engines. The break-up and mixing processes are, however, not well understood. In the pursuit to address this problem, the past decade has seen the appearance of several new experimental techniques that have successfully provided quantitative information on the break-up in the dense spray region [1], including ballistic imaging (BI). BI is a line-of-sight optical measurement technique providing high-resolution images of structures buried inside spray regions inaccessible with conventional imaging techniques. This diagnostic is commonly implemented using short pulse (e.g. $\sim 100$ fs pulse widths) lasers and low light level imaging systems. The application of time-gated BI (TGBI) for spray studies has recently been reviewed in detail by Linne [1]. The technique relies on multiply scattered off-axis photons being rejected by a very fast $(\sim 1.5 \mathrm{ps})$ optical Kerr effect (OKE) time gate. This allows transmission of early arriving, useful imaging light while rejecting the later photons to produce a single-shot ballistic image. Examples of two optical setups are shown in Fig. 1. The main difference between the two setups is in how the image is propagated to the camera. The setup in Fig. 1a, here called the $4 f$ setup, is similar to the arrangement used in earlier work by Alfano et al. [2], and it matches the setup used in early spray studies [3]. The setup in Fig. 1b, here called the $2 f$ setup, was first used in spray measurements by Schmidt et al. [4]. Both configurations have been used to provide single-shot images of near-nozzle breakup but they have not been compared experimentally. In this work, we compare the contrast transfer functions (CTF) of the two different setups using experimental data obtained under a series of scattering conditions. To further investigate differences between the two setups, alignment sensitivity of both optical systems were modeled using a physical optics code.

\section{Experiments}

The CTF of each configuration was measured by imaging a resolution test chart through a series of scattering conditions designed to replicate those of typical Diesel sprays issuing into room-temperature air. The scattering environment was simulated with a monodisperse suspension of varying sizes and concentrations of polystyrene (PS) spheres in distilled water. Typical Diesel spray droplet sizes, depending on conditions, fall in the range of 5-20 $\mu$ m [5]. Thus, our measurements are analogous to $5,10,15$, and $20 \mu \mathrm{m}$ Diesel droplets in varying concentrations. The corresponding PS sphere sizes were determined by matching the Mie scattering phase functions of available PS spheres with the Diesel droplets. This matching procedure resulted in the selection of the following PS sphere sizes: 3.5, 8.0, 9.7, and 14.9 $\mu \mathrm{m}$. Optical depth (OD) was used to quantify the various concentrations investigated. In accordance with arguments by Linne [1], the number of scattering events was equated to OD (they are roughly similar for this size range [6]); OD ranged from 0 to 14 .

The experimental configurations are shown in Fig. 1. The beam from a $1 \mathrm{kHz}$ repetition rate chirped-pulse regenerative amplifier (Spectra-Physics Spitfire) providing $180 \mathrm{fs}, 4 \mathrm{~mJ}$ pulses centered at $800 \mathrm{~nm}$ (beam diameter of $8 \mathrm{~mm}$ ) was split into an imaging beam and a gating beam. The gating beam was used to activate birefringence in the carbon disulfide $\left(\mathrm{CS}_{2}\right)$, creating a gate time $\sim 1.5 \mathrm{ps}$ FWHM. After the beam splitter, the imaging beam passed through a 
polarizer/wave plate combination providing polarization and power control. The cell containing the PS sphere suspension, with the resolution test chart embedded in the center, was placed directly after this first polarizer. The image was projected onto a frosted glass screen and captured with an Andor iXon 885 EM-CCD camera. All images were background subtracted and flat-field corrected. In the $4 f$ configuration (Fig. 1a) the object plane is placed one focal length

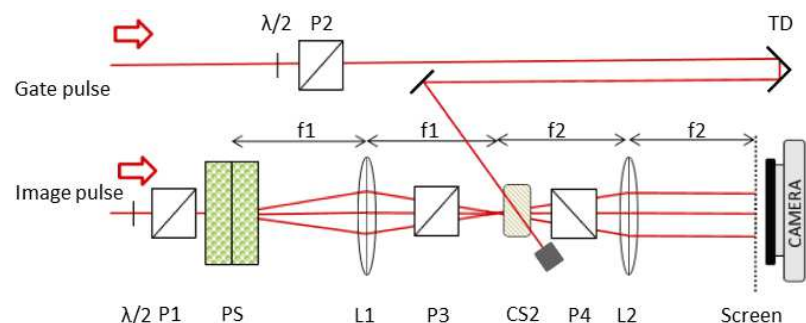

(a) $4 f$ setup

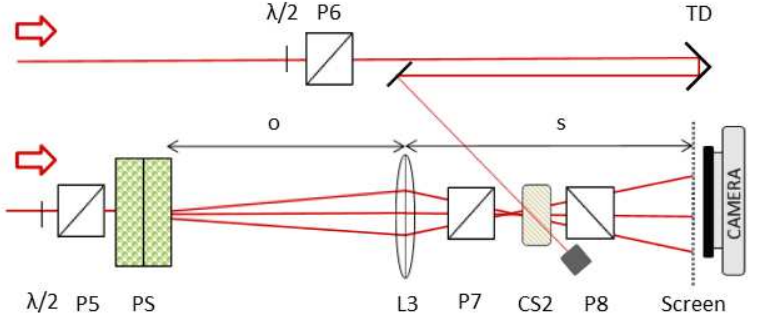

(b) $2 f$ setup

Fig. 1: Optical setups. Symbols: $\lambda / 2$ : half wave plate, P1-P8: $15 \times 15 \mathrm{~mm}^{2}$ aperture Glan-Laser polarizers, L1-L3: achromatic lenses, CS2: $40 \mathrm{~mm}$ aperture, $10 \mathrm{~mm}$ path length $\mathrm{CS}_{2}$ container, TD: time delay, $f_{1}-f_{2}$ focal lengths for the respective lens, o and s: object-to-lens and lens-to-image distances respectively.

in front of the collecting lens and the image plane is located one focal length behind the last lens. The OKE-gate is placed between the two lenses with the $\mathrm{CS}_{2}$ cell slightly behind the focal point of the collecting lens to avoid focusing directly into the $\mathrm{CS}_{2}$. The lenses used in this configuration were 3" diameter achromatic doublets with focal lengths of $f_{1}=250 \mathrm{~mm}$ and $f_{2}=500 \mathrm{~mm}$ yielding an image magnification of two and a collection half angle of $8.7^{\circ}$ degrees. The $2 f$ setup (Fig. 1b) uses a single lens to generate a real image of the object at the screen. We attempted to match magnification and collection angle of the two configurations as closely as possible. Using a 2" achromatic doublet lens with $f_{3}=150 \mathrm{~mm}$ placed $225 \mathrm{~mm}$ behind the test chart object resulted in an image magnification of two and a collection half angle of $6.4^{\circ}$ degrees.

\section{Results and Discussion}

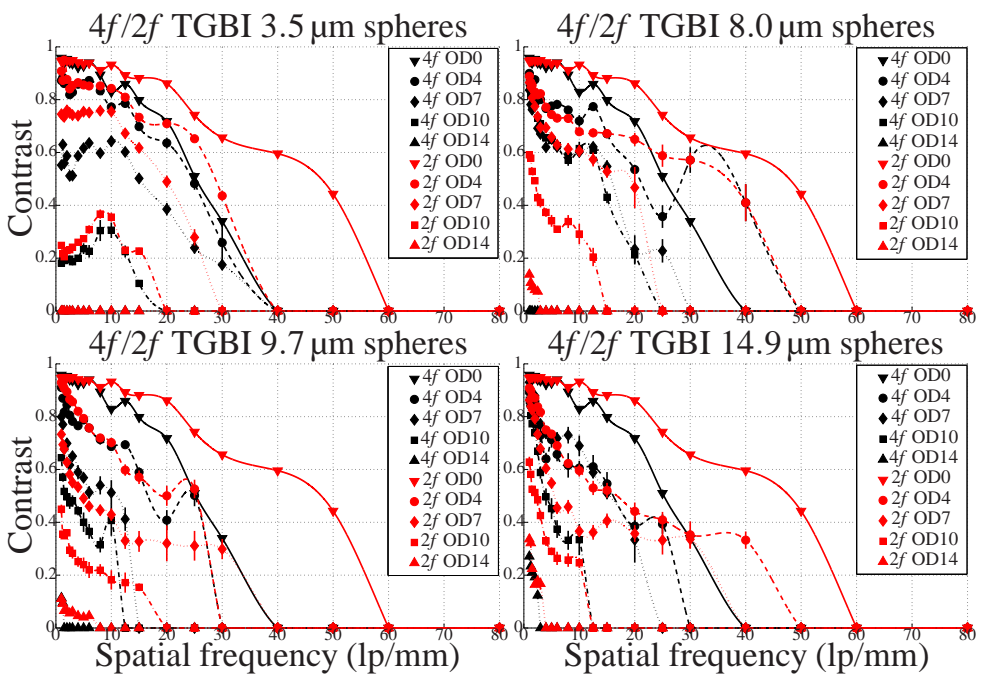

Fig. 2: Experimentally measured CTFs for $4 f$ and $2 f$ TGBI

The experimental results are shown in Fig. 2. In the OD0 case, which was achieved using pure distilled water, the $4 f$ and $2 f$ setups exhibited ultimate resolutions of $30 \mathrm{lp} / \mathrm{mm}$ and $50 \mathrm{lp} / \mathrm{mm}$ respectively. The $2 f$ setup had notably better image resolution before the introduction of scatterers. With the introduction of scatterers, the data shows the highest 
resolvable spatial frequency is similar for both setups for all ODs and sphere sizes. The $2 f$ setup appears to show higher absolute contrast in the lower end of the sphere size spectrum and in the densest cases. With larger sphere sizes at moderate ODs the results are mixed. When looking at oscillations in the CTFs, which are an indication of system aberrations, one sees that they are more pronounced in the $4 f$ case.

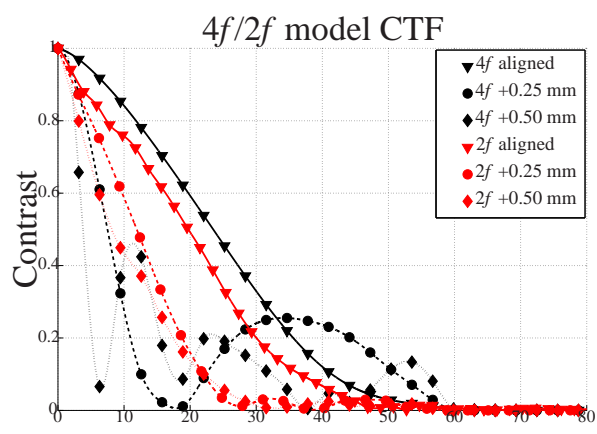

Spatial frequency $(\mathrm{lp} / \mathrm{mm})$

Fig. 3: Modeled CTFs as function of object to collecting lens distance for $4 f$ and $2 f$ setups.

To further investigate alignment sensitivity of the two setups, both were analyzed using GLAD, a commercially available physical optics code. This simulation included the lenses and the polarizers in order to examine the effect of the imaging optics. A diverging Gaussian beam with a $4 \mu \mathrm{m}$ radius was propagated through the setups. In the image plane, a pseudo point spread function was extracted and a CTF could be constructed. Due to the finite extent of the object plane light source, this CTF is not directly comparable to experimental data. When comparing system responses, however, it gives a valid representation of effects due to misalignments. Responses due to transverse and rotational misalignment of the collecting lens and misalignment in the depth-of-field were investigated. In the three tested cases, the $4 f$ was more sensitive to misalignment than the $2 f$. The results for misalignment along the optic axis are shown in Fig. 3. The responses to transverse and rotational misalignment show similar behavior.

When considering tendency to introduce aberrations, the $2 f$ setup appears to be preferable. Both the experimental and simulated data show that it is less prone to aberrations due to optical misalignments. It is less sensitive to alignment precision and has a longer depth of field. These are two desirable characteristics for imaging systems intended for use in the adverse conditions of transient combustion and spray measurements. An investigation into mechanisms causing the experimentally measured aberrations is ongoing. The work was funded by the Swedish Research Council.

\section{References}

1. M. Linne, "Imaging in the optically dense regions of a spray: A review of developing techniques," Progress in Energy and Combustion Science 39, 403-440 (2013).

2. L. Wang, P. P. Ho, C. Liu, G. Zhang, and R. R. Alfano, "Ballistic 2-d imaging through scattering walls using an ultrafast optical kerr gate," Science 253, 769-771 (1991).

3. M. Paciaroni and M. Linne, "Single-shot, two-dimensional ballistic imaging through scattering media," Appl. Opt. 43, 5100-5109 (2004).

4. J. Schmidt, Z. Schaefer, T. Meyer, S. Roy, S. Danczyk and J. Gord, "Ultrafast time-gated ballistic photon imaging and shadowgraphy in optically dense rocket sprays," Appl. Opt. 48, B137-B144 (2009).

5. J. Labs, "Droplet size and volume fraction in the near-injector centerline region of diesel sprays," In: ILASSAmericas, 16th Annual Conference on Liquid Atomization and Spray Systems, Monterey, California, U.S. (2003).

6. E. Berrocal, "Multiple scattering of light in optical diagnostics of dense sprays and other complex turbid media." Ph.D. thesis, School of Engineering, Cranfield University, Cranfield, UK (2006). 